\title{
Study on the Applicability of Tyvek in Electric Bicycle Riding Protective
}

\author{
Lu Lin ${ }^{\mathrm{a}, 1}$, Jiayi Qiu ${ }^{\mathrm{a}}$, Yu Wang a and Zhangyi Lin ${ }^{\mathrm{a}}$ \\ ${ }^{a}$ Art and Design Institute, Zhejiang Sci-Tech University, Hangzhou, China, 310018
}

\begin{abstract}
This paper aims to introduce Tyvek into the existing fabrics of electric bicycles (EBs) protection articles, and demonstrate its feasibility. We did our research based on the protection needs of users during transport. The study carried out experimental research from the perspective of safety assessment and comfort assessment combined with relevant ISO and Chinese national evaluation standards to verify the feasibility of the materials. This article outlines the experimental method, such as wet wash resistance test, wear resistance test elated to safety, and air permeability test, water-vapour transmission test and UV resistance test related to comfort were carried out. In the end we discuss future developments.
\end{abstract}

Keywords. Tyvek, electric bicycle, material study, EBs protective equipment

\section{Introduction}

In recent years, with the shortage of fossil fuels and other resources and the increasingly serious problem of environmental pollution, new energy vehicles have attracted more attention from the international community. Among them, electric bicycles (EBS) have attracted more attention because of their lightness and flexibility. From 2012 to 2014, the global production of electric two wheeled vehicles increased significantly from 28 million to 40 million [1]. With the increase of market share, personal travel protection needs tend to be diverse, and users have higher safety and comfort requirements for the original riding windproof and rainproof products in terms of product function and product form. Compared with the original products, we hope to explore more possibilities for innovation from the perspective of materials [2].

Tyvek material is a recyclable environmental protection material, which has two structural materials of "hard" and "soft". It is widely used in the fields of protection [3], planting [4], medical packaging, clothing and packaging because of its waterproof, tear resistant, degradable and UV resistant properties. In the consumer market, the products of Tyvek materials are deeply loved by consumers with the label of "young and fashionable". Therefore, the application fields of Tyvek materials are more and more extensive. Starting from the user's use feeling, this experiment analyzes whether the basic performance of Tyvek material meets the basic requirements of electric riding, meets the user's use feeling and brings the possibility of innovation to the design through safety test and comfort test.

\footnotetext{
${ }^{1}$ Corresponding author: Lu Lin; Postal address: Art and Design Institute, Zhejiang Sci-Tech University,
} Hangzhou, China, 310018 ; Email address: vichy0723@126.com 


\section{Design Concepts}

In this section, we will introduce two types of electric riding needs. These concepts are based on the results of our preliminary survey and Maslow's hierarchy of needs [5].

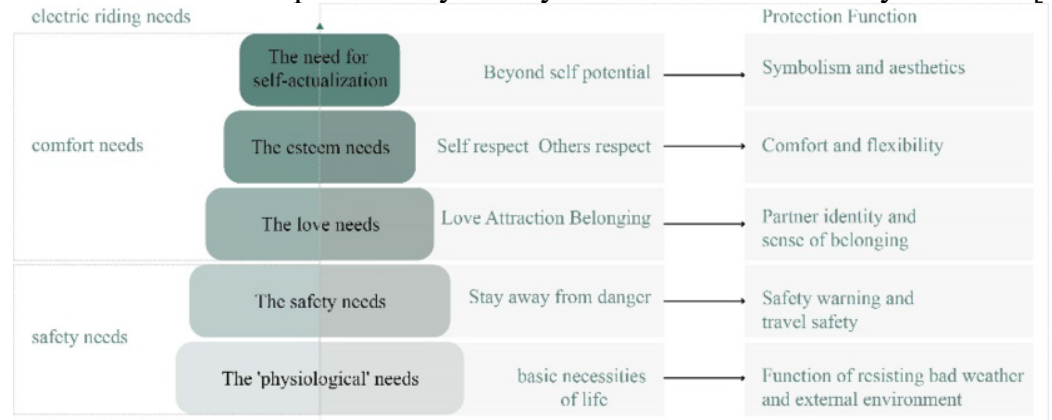

Figure 1. Application of demand analytic hierarchy process in EBs riding.

\subsection{Safety Requirement}

Safety \& security needs in Maslow's hierarchy of needs theory refers to the need to prevent itself from being endangered [6]. The free and flexible characteristics of EBs bring many uncertain factors to users. Because electric riders are exposed to the outdoor environment for a long time during riding, they put forward certain requirements for the protection function in the travel scene. At present, EBs protective equipment mainly meets the basic needs of users, can ensure users to resist the external climate or environment and reduce physical injury when riding, which is also the premise of EBs protective products to diversified design and research. In the hierarchy of needs theory, only by meeting the physiological needs of users, users can put forward needs at a higher level, so as to truly optimize users' use feeling. Therefore, protective equipment should ensure the safety needs of users when using EBs in traffic environment, such as providing warning and reminder function in combination with reflective stickers.

\subsection{Comfort Requirement}

When users' security needs are met, they will have the needs of love and respect. On the one hand, it enables users to feel the love from products and make themselves full of confidence and achievements; The second is to be respected by others, that is, to obtain the recognition and appreciation of others [6]. In EBs protective equipment, it refers to the experience between users and products, and users can get a good experience through the use process. This puts forward higher requirements for the durability and comfort of protective articles. 


\section{Method}

\subsection{Preparation}

As far as our research is concerned, this study is divided into two stages: safety test and comfort test. Before the test, we first investigated EBs protection products in the current Chinese market, and finally selected the common external fabrics in the category of weather proof products, such as PVC waterproof cloth, PU, Oxford cloth, and four Tyvek materials for comparative experiment.

- $\quad$ PVC waterproof cloth: the polyester surface is coated with PVC coating to make its surface smoother, with good waterproof performance and low cost. However, PVC waterproof cloth is easy to fall off and reduce its functionality.

- PU fabric: PU is a coating based on fabric. Polyurethane film is coated on its surface and heated and pressurized to form a composite material. Compared with PVC fabric, Pu has good softness, elasticity, twists and turns resistance and good air permeability.

- Oxford cloth: Oxford cloth is a fabric interwoven with a variety of chemical fibers. It has the characteristics of easy washing and quick drying, loose and dry feel and good moisture absorption. It is treated by two-layer surface processing process of silver coating. It has the properties of waterproof, sunscreen and durability. Compared with PVC fabric and Pu fabric, Oxford fabric lacks elasticity.

Tyvek material is a degradable and recyclable material. It is a non-woven technology material made of $100 \%$ high-density polyethylene by flash spinning and bonding process. It has the properties of waterproof, breathable, light weight, toughness, tear resistance, bacteria prevention, dust prevention, UV resistance and so on. It is widely used in various fields. We combine the commonly used Tyvek models with the design requirements of EBS protective articles, and select the models with a thickness of less than $0.2 \mathrm{~mm}$ as the samples of this experiment: 1025D, 1056D, 1443R, 1433R with PU.

- 1443R: soft and light touch, with regular concave convex dot arrangement on the surface.

- $\quad 1433 \mathrm{R}$ with Pu: Tyvek surface is coated with a layer of PU. The PU surface is smooth and the reverse side is rough and dry. Compared with the soft and thin 1433R, the surface has better elasticity and hardness.

- 1025D: weight and thickness are less than 1443r, but more rigid.

- 1056D: it is the model with the largest weight and thickness among all samples, and the tactility of the material is more stiff and smooth than $1025 \mathrm{D}$.

\subsection{Experiment Process}

Firstly, we conduct safety test from the perspective of users' safety requirements. The purpose of the experiment is to hot stamp the safety warning on the material surface through the hot stamping process. In order to reduce the falling off of safety warnings, it is necessary to coordinate the hot stamping temperature and the temperature resistance of Tyvek materials during the test, and determine the best hot stamping temperature of Tyvek materials. It is also necessary to determine the firmness of the safety warning strip on the surface of Tyvek material through water washing test and wear performance test. The purpose of water washing performance test is to conduct washing at different times 
and for a long time through the washing machine, observe the falling off degree of reflective stickers on the surface of Tyvek materials, and conduct four tests in a time period of 38 minutes to determine the firmness of reflective stickers in Tyvek materials. The purpose of the wear performance test is to take the wool felt as the corresponding wear object for 100 times, 300 times, 500 times, 700 times, 900 times, 1100 times, 1300 times and 1500 times respectively, and further analyze the firmness of the reflective sticker in Tyvek material.

The comfort test is mainly carried out to meet the waterproof, sunscreen, windproof and other needs of users when using protective products. The experiments include air permeability [7], water-vapour transmission and UV resistance. The purpose of the air permeability test is to derive the wind protection performance of the material through data analysis. The water-vapour transmission test is to compare and analyze the water resistance of materials by observing their moisture resistance in an environment with $90 \%$ humidity. The aim of UV-resistant experiments is to deduce the UV-resistant properties of materials by observing their surface changes under accelerated UV irradiation. Tyvek and other materials are graded according to their performance comparison and the Chinese trade standard "Evaluation of wind and moisture resistance of textiles". Finally, the best material is selected by combining the experimental results.

\section{Results And Discussion}

\subsection{Safety test}

In safety experiments, the first step is to bond the Tyvek material to the safety warning strip. In Tyvek materials, the main bonding methods are printing, sticking, sewing, heatsealing, etc. In the processing of reflective materials, there is a thermal transfer process which scalds the reflective pattern on the surface of the fabric by heating it. Compared with sewing and sticking methods, thermal transfer printing is easy to operate, fast and has bright coloring effect, which provides a fast process for safety warning strip. Therefore, we first attempt to apply the heat transfer process to the processing of safety warning strips.

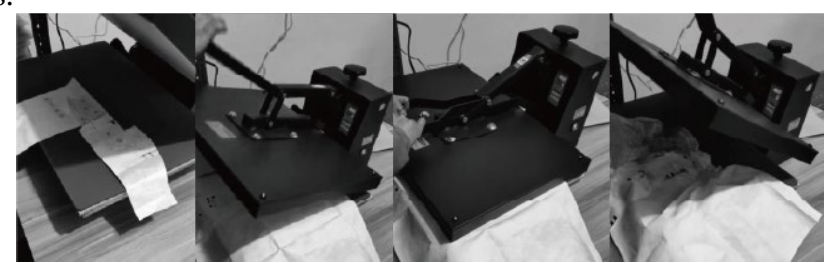

Figure 2. Preparation of safety test.

Table 1. The result of safety test.

\begin{tabular}{ccl}
\hline Temperature & State & Tyvek \\
\hline $160^{\circ} \mathrm{C}$ & Melt & $1433 \mathrm{R}$ \\
& & $1433 \mathrm{R}$ with PU \\
& & $1025 \mathrm{D}$ \\
& & $1056 \mathrm{D}$ \\
& & None \\
$130^{\circ} \mathrm{C}$ & Unmelted & $1433 \mathrm{R}$ \\
& Melt & $1433 \mathrm{R}$ with PU \\
& & $1025 \mathrm{D}$ \\
& & $1056 \mathrm{D}$
\end{tabular}


As the temperature of ordinary scalding process is $130{ }^{\circ} \mathrm{C}-160{ }^{\circ} \mathrm{C}$ and the melting point of Tyvek is $135^{\circ} \mathrm{C}$, the scalding temperature is almost close to the melting point. We have adjusted the temperature unit for one pressing at $10{ }^{\circ} \mathrm{C}$ and found that Tyvek still melts when it drops to $130{ }^{\circ} \mathrm{C}$. Finally, we press the Tyvek surface with positioning film at $130{ }^{\circ} \mathrm{C}$ so that the temperature of the embossing pattern is higher than that of Tyvek. It can not only press the reflective strip but also make Tyvek fail to reach the melting temperature. Therefore, the pattern can be successfully pressed on the Tyvek surface. During the experiment, 1433R, 1433R with PU and 1025D shrink sharply even when they are transferred with positioning film. Therefore, only $1056 \mathrm{D}$ with $130^{\circ} \mathrm{C}$ (positioning film) was processed into the washability test in this experiment.

\subsubsection{Wet wash resistance test}

Press Tyvek 1056D with a safety warning strip before the experiment. Three of the samples were used as test groups and washed in a washer. Set the washing time to 38 minutes for a period of time, taking out one sample every 38 minutes until all four samples are removed. Untreated samples as control group were compared with three water-washed samples to observe the water-washed condition of each sample. Secondly, the sample is illuminated by lamp to analyze the reflectance after washing.

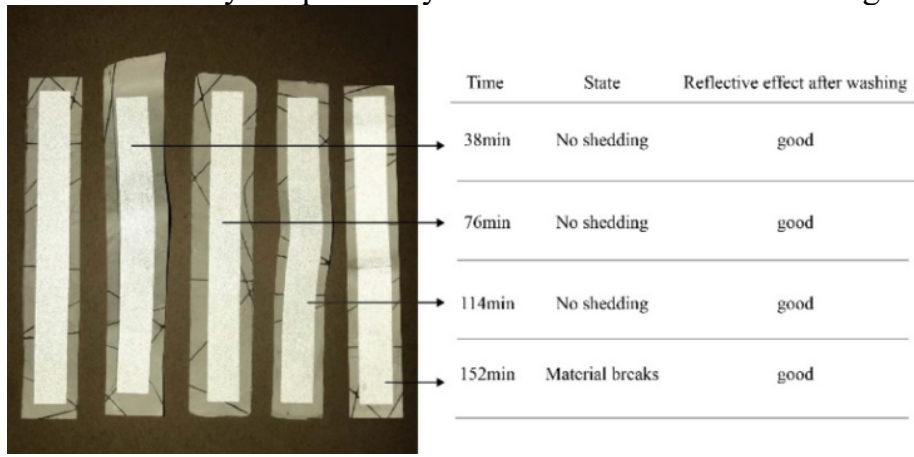

Figure 3. Comparison of reflective strip effect under different washing time.

It can be seen from the wash condition table for four periods that the safety warning strip does not fall off and the reflectance after wash is the same as that of untreated samples. In addition, some safety warning strips were bent in the base material during the washing process, which led to the appearance of faults locally in the safety warning pattern and slight shedding at the faults. It indicates that the safety warning strip needs to be based on a curved surface which will not bend in order to reduce the phenomenon of water washing off.

\subsubsection{Wear resistance test}

First, prior to the beginning of the experiment, we prepared the material of the analogue fabric wear tester: cylindrical $5 \mathrm{~cm}$ in diameter and wool felt $10 \mathrm{~cm}$ in diameter as wear material. Next, prepare three $310 \mathrm{~mm} * 100 \mathrm{~mm}$ rectangular specimens and fix them on the bottom plate in turn. During the experiment, the wear force remains the same as possible, reducing errors caused by human factors. The direction of friction rubs repeatedly in a circular position. Photographs are taken every 200 times to record wear. Finally, the table status of all samples is analyzed and summarized. 
Table 2. Wear resistance test of reflective strip.

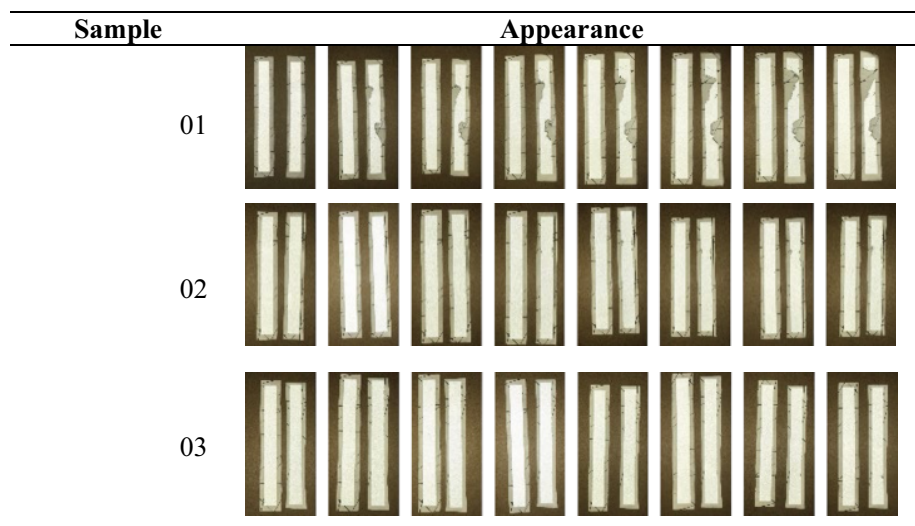

the number of frictions from left to right is: 100300100 300500700900110013001500

During the wear property test, sample 01 shows two areas falling off at 300 times of wear and then extends along both notches between 300 and 1500 times. When sample 01 is not beginning to wear out, two protrusions appear in the folds of the material itself, which may be responsible for the rapid shedding of the reflector strip. When sample 02 is worn, the surface of sample 02 is smooth and slightly crimped on the rectangular edge at 900 times of wear until 1500 times of wear, the surface of sample 02 is still in a relatively complete state. Sample 03 did not fall off under 0 - 1500 wear cycles. It can be inferred from the wear degree of the three samples that the reflective sticker can be fixed on the surface of Tyvek material by hot-stamping process, but the surface of Tyvek material must be smooth and unreasonable, otherwise the reflective warning pattern will not be pressed uniformly and fall off.

\subsection{Comfort test}

The sample materials for the three groups of comfort tests were: PVC waterproof cloth, PU, Oxford cloth, 1056D, 1433R, 1025D, 1433R with PU.

\subsubsection{Air permeability test}

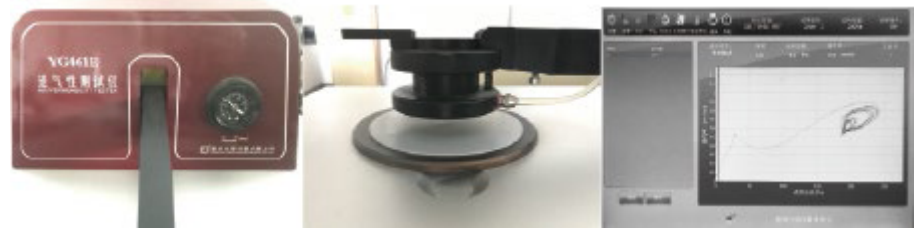

Figure 4. Experiments were performed using a YG461D digital fabric air permeability instrument.

Prepare $20 \mathrm{~cm}^{2}$ sample before experiment and cut into specified shape. For the air permeability meter, set the pressure differential to $200 \mathrm{pa}$ according to the industrial fabric. Next, the sample is placed on the test stand and formally tested. Wait 1-2 minutes during the experiment until the permeability meter reads the valid permeability and averages each sample 10 times in different directions. When all the samples have been tested, the air permeability of the existing EBs protection products' material is calculated according to GB8170, and the value of the air permeability area is determined to screen the four Tyvek materials. The equation for the test is: 


$$
R=\frac{q v}{A} * 167 \quad(\mathrm{~mm} / \mathrm{s})
$$

where qv is the arithmetic mean flow rate of air, in cubic decimeters per minute $\left(\mathrm{dm}^{3} / \mathrm{min}\right.$, $1 / \mathrm{min})$; $\mathrm{A}$ is the area of fabric under test, in square centimeters $\left(\mathrm{cm}^{2}\right) ; 167$ is the conversion factor from cubic decimeters (or liters) per minute per square centimeter to millimeters per second $\left(\mathrm{dm}^{3} / \mathrm{cm}^{2}, 1 / \mathrm{cm}^{2}\right)$. [7]

Table 3. Air permeability test results for seven materials.

\begin{tabular}{rrrrrrrrrrrrr}
\hline Sample & $\mathbf{1}$ & $\mathbf{2}$ & $\mathbf{3}$ & $\mathbf{4}$ & $\mathbf{5}$ & $\mathbf{6}$ & $\mathbf{7}$ & $\mathbf{8}$ & $\mathbf{9}$ & $\mathbf{1 0}$ & \multicolumn{1}{c}{$\boldsymbol{v}$} & $\mathbf{R}$ \\
\hline 1056D & 2.85 & 1.51 & 1.70 & 1.24 & 1.83 & 1.50 & 1.59 & 1.67 & 2.04 & 1.69 & 1.76 & 0.73 \\
$1025 \mathrm{D}$ & 2.36 & 2.56 & 2.32 & 2.06 & 1.89 & 1.82 & 2.07 & 1.78 & 2.28 & 2.56 & 2.17 & 0.91 \\
$1433 \mathrm{R}$ with & 0.24 & $/$ & $/$ & $/$ & 0.28 & $/$ & $/$ & 0.31 & $/$ & $/$ & 0.27 & 0.11 \\
$\mathrm{Pu}$ & & & & & & & & & & & & \\
$1433 \mathrm{R}$ & 0.70 & 1.02 & 0.77 & 0.72 & 0.78 & 0.98 & 0.80 & 0.81 & 1.30 & 1.20 & 0.908 & 0.38 \\
PVC & 0.37 & 0.52 & 0.72 & 0.43 & 0.45 & 0.57 & 1.37 & 0.64 & 0.61 & 0.40 & 0.6 & 0.25 \\
PU & 3.9 & 3.91 & 3.76 & 3.82 & 4.00 & 4.27 & 4.74 & 5.05 & 4.84 & 4.81 & 4.31 & 1.80 \\
Oxford & 74.5 & 76.7 & 71.7 & 71.5 & 75.1 & 67.2 & 79.2 & 76.6 & 75.4 & 74.5 & 74.27 & 31.00 \\
cloth & 0 & 1 & 0 & 9 & 6 & 3 & 0 & 2 & 8 & 0 & & \\
\hline
\end{tabular}

The air permeability of these seven samples are summarized in Table 3. The result of most of the samples is less than or equal to $10 \mathrm{~mm} / \mathrm{s}$, and they have better wind resistance and belong to the third class. The permeability of Oxford cloth is $31 \mathrm{~mm} / \mathrm{s}$, which belongs to the second-order sample. The order of air permeability from small to large is $1443 \mathrm{R}$ with $\mathrm{PU}>\mathrm{PVC}$ waterproof cloth $>1433 \mathrm{R}>1056 \mathrm{D}>1025 \mathrm{D}>\mathrm{PU}$ fabric $>$ Oxford cloth. In the air permeability test, $1433 \mathrm{R}+\mathrm{PU}$ cannot get the air permeability because of its small air permeability. Many times, the data are close to zero, which also shows that the material has poor air permeability and best wind resistance. Oxford cloth is the best test material for air permeability, the better the air permeability, the worse the wind resistance.

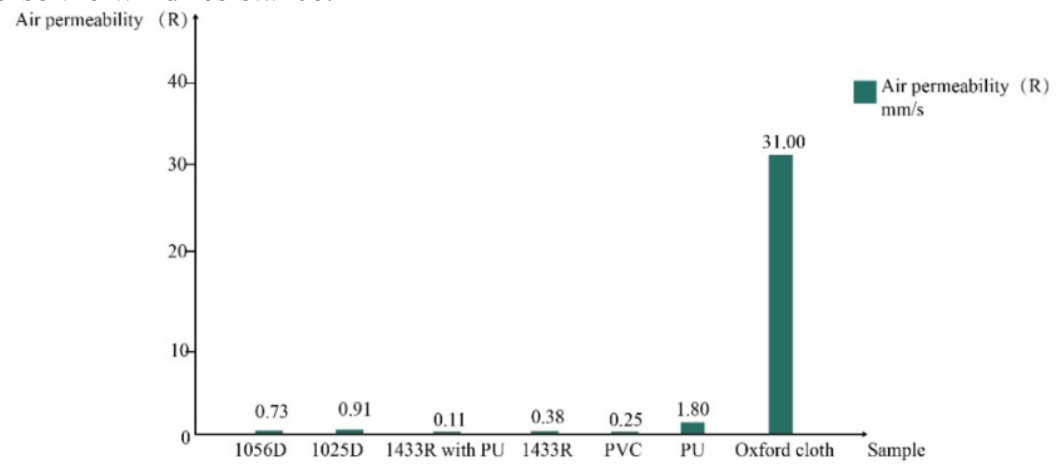

Figure 5. Bar chart for Air Permeability Comparison.

According to China industry standards [8], the above samples are all third-class except Oxford cloth (with excellent wind and moisture resistance). The air permeability of PU fabric is $1.80 \mathrm{~mm} / \mathrm{s}$, which means good wind resistance. The air permeability of Oxford cloth is $31.00 \mathrm{~mm} / \mathrm{s}$ and higher, which means poor wind resistance, as shown in the figure. Therefore, all Tyvek models tested in this test are suitable for manufacturing wind and moisture proof protective products.

\subsubsection{Water-vapour transmission test}

The experimental materials ware: $7 \mathrm{~cm}^{2} \mathrm{PVC} * 1,7 \mathrm{~cm}^{2} \mathrm{PU} * 1,7 \mathrm{~cm}^{2}$ Oxford cloth $* 1,7 \mathrm{~cm}^{2}$ $1056 \mathrm{D} * 1,7 \mathrm{~cm}^{2} 1433 \mathrm{R} * 1,7 \mathrm{~cm}^{2} 1025 \mathrm{D} * 1,7 \mathrm{~cm}^{2} 1433 \mathrm{R}$ with $\mathrm{P} \mathrm{U}^{*} 1$. The experimental 
equipment includes: oven, YG501D water-vapour transmission test box, water-vapour transmission cup, moisture absorbent, silicone dryer, electronic scale.
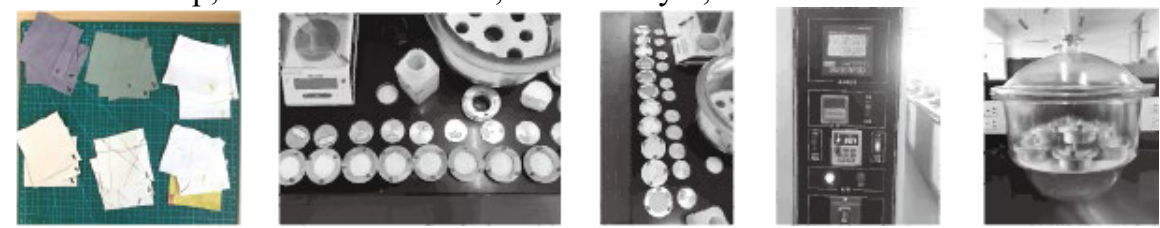

Figure 6. Experiments were performed using a YG501D water-vapour transmission test box.

We choose the desiccant method in the performance test of the water-vapour transmission method. Dry anhydrous calcium chloride in an oven at $160^{\circ} \mathrm{C}$ for 3 hours before the experiment, then take it out and pour it into a moisture-permeable cup. Three specimens are taken from each sample to mark the test surface and the specimens are fixed to $2 \mathrm{~mm}-3 \mathrm{~mm}$ above anhydrous calcium chloride. Secondly, the humidity and temperature of the water-vapour transmission test box are preset. Temperature and humidity are set at $38^{\circ} \mathrm{C}$ and $90.0 \%$. The humidity in the iso-permeability test box is kept in balance for 1 hour. Put the uncovered sample into the box for half an hour and quickly remove it into the silica gel dryer for 30 minutes. Finally, put it into the electronic scale and call it the data of full integration of sample and water vapor. Then, put the sample back in the humidification test box for 1 hour, take out and put it in the silica gel dryer for half an hour, and the data as shown in Table 4. The equation for the test is:

$W V T=(24 \cdot \Delta m) /(S \cdot t)$

where WVT is daily (24h) water-vapour transmission rate per square meter, in square meters per day (g/ (m2.d)); $\Delta m$ is the difference between two weighing of the same test $(\mathrm{g})$; $\mathrm{S}$ is the area of fabric under test, in square meters $\left(\mathrm{m}^{2}\right)$; $\mathrm{t}$ is the test time $(\mathrm{h})$.

Table 4. Water-vapour transmission test results for seven materials.

\begin{tabular}{|c|c|c|c|c|c|}
\hline Sample & & $\begin{array}{c}\text { Initial } \\
\text { weight } \\
\text { (g) }\end{array}$ & $\begin{array}{c}\text { Weight after } 1 \text { hour } \\
(\mathrm{g})\end{array}$ & $\begin{array}{c}\text { Mean weight } \\
\text { (g) }\end{array}$ & $g /\left(m^{2} \cdot d\right)$ \\
\hline \multirow[t]{3}{*}{ 1433R } & 01 & 199. 959 & 200.534 & \multirow{3}{*}{0.513} & \multirow[t]{3}{*}{3198} \\
\hline & 02 & 199. 907 & 200.320 & & \\
\hline & 03 & 200.241 & 200.851 & & \\
\hline \multirow[t]{3}{*}{$1056 \mathrm{D}$} & 01 & 198. 588 & 199.178 & \multirow[t]{3}{*}{0.574} & \multirow[t]{3}{*}{3444} \\
\hline & 02 & 198. 581 & 199.152 & & \\
\hline & 03 & 197. 583 & 198.143 & & \\
\hline \multirow[t]{3}{*}{$1025 \mathrm{D}$} & 01 & 163. 214 & 163.841 & \multirow[t]{3}{*}{0.615} & \multirow[t]{3}{*}{3690} \\
\hline & 02 & 165.330 & 165.896 & & \\
\hline & 03 & 165.586 & 166.239 & & \\
\hline \multirow{3}{*}{$\begin{array}{l}\text { 1433R } \\
\text { with } \mathrm{Pu}\end{array}$} & 01 & 202. 839 & 202.961 & \multirow[t]{3}{*}{0.164} & \multirow[t]{3}{*}{984} \\
\hline & 02 & 204. 174 & 204.412 & & \\
\hline & 03 & 201. 266 & 201.399 & & \\
\hline \multirow[t]{3}{*}{ PVC } & 01 & 165. 286 & 165.404 & \multirow[t]{3}{*}{0.083} & \multirow[t]{3}{*}{498} \\
\hline & 02 & 165.893 & 165.958 & & \\
\hline & 03 & 167. 065 & 167.131 & & \\
\hline
\end{tabular}




$\begin{array}{cccccc}\text { PU } & 01 & 200.735 & 201.009 & 0.255 & 1530 \\ & 02 & 196.628 & 196.870 & & \\ \text { Oxford } & 03 & 194.570 & 194.819 & & 5448 \\ \text { cloth } & 01 & 167.166 & 168.094 & 0.908 & \\ & 02 & 167.988 & 168.857 & & \\ & 03 & 164.198 & 165.125 & & \end{array}$

The water-vapour transmission rate of the EBs protection products' material is quite different. Oxford cloth has good water-vapour transmission rate in the EBs protection products' material (it is a comfortable feeling to contact with human body). The PVC waterproof cloth has the smallest value in water-vapour transmission rate, and users will feel stuffy during use. Secondly, the water-vapour transmission rate of different Tyvek samples is relatively stable, all above $3000 \mathrm{~g} /\left(\mathrm{m}^{2} . \mathrm{d}\right)$, the order from big to small is: $1025 \mathrm{D}>1056 \mathrm{D}>1433 \mathrm{R}>1433 \mathrm{R}+\mathrm{PU}$, which is within the water-vapour transmission rate value of EBs protection products' material.

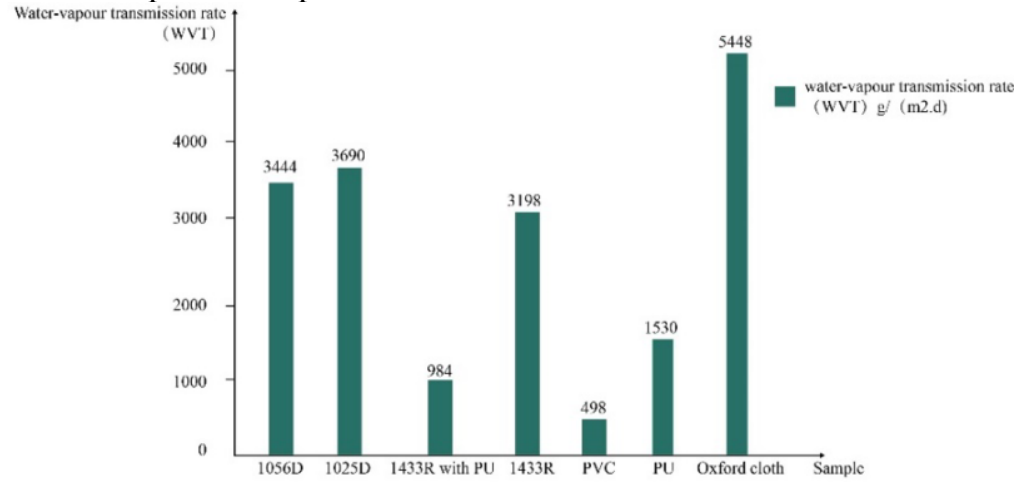

Figure 7. Bar chart for water-vapour transmission comparison.

GB/T 21980-2008 requires a water-vapour transmission rate of more than or equal to $2500 \mathrm{~g} /\left(\mathrm{m}^{2} . \mathrm{d}\right)$ for protective articles [9]. It is known from the material of the windscreen that the water-vapour transmission rate of both the PVC waterproof cloth and the PU fabric is less than $2500 \mathrm{~g} /\left(\mathrm{m}^{2} . \mathrm{d}\right)$ does not meet the requirements for watervapour transmission rate of protective articles. Oxford cloth meets the specifications for protective articles, but Oxford cloth has high air permeability and poor wind resistance. As shown in Figure 7. In different types of Tyvek materials, the water-vapour transmission rate index of materials is more than $2500 \mathrm{~g} / \mathrm{s} .\left(\mathrm{m}^{2} . \mathrm{d}\right)$, which meets the requirements of protective articles for water-vapour transmission rate. However, the value of water-vapour transmission rate of Tyvek model compounded with PU after later period is $984 \mathrm{~g} /\left(\mathrm{m}^{2} . \mathrm{d}\right)$, and the water-vapour transmission rate is less than 2500 , which does not meet the standard of general technical specifications for protective articles.

\subsubsection{UV resistance test}

The experimental materials ware: $7 \mathrm{~cm} * 5 \mathrm{~cm} \mathrm{PVC} * 1,7 \mathrm{~cm} * 5 \mathrm{~cm} \mathrm{PU} * 1,7 \mathrm{~cm} * 5 \mathrm{~cm}$ Oxford cloth *1, 7cm*5cm 1056D*1,7cm*5cm 1433R*1, 7cm*5cm1025D*1, 7cm² 1433R with $\mathrm{P} \mathrm{U}^{*} 1$. The experimental equipment is QUV/spray (accelerated weathering tester).

Before the experiment, cut $5 \mathrm{~cm} * 7 \mathrm{~cm}$ rectangular sample according to the specified size, fix the square sample on the clamping plate and put it into the UV aging tester to simulate the UV light conditions in natural climate. Take out the sample by accelerating 
ultraviolet radiation for four hours. Place the sample in an oven at $50^{\circ} \mathrm{C}$ and let it stand for four hours to remove. Observe the illuminated sample and the illuminated sample to compare and analyze, and select the best sample with better UV resistance.

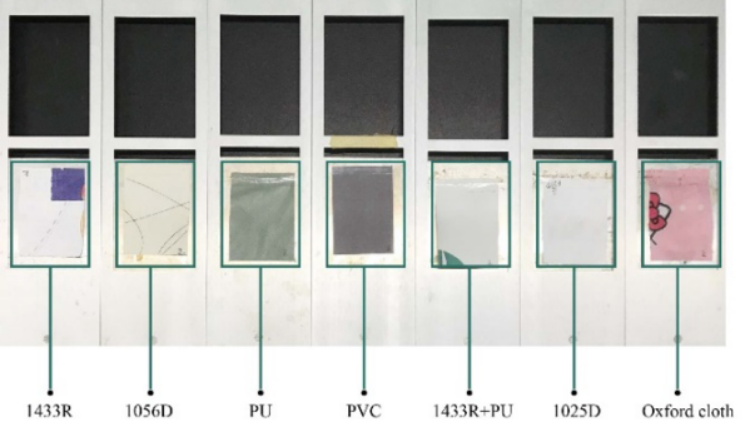

Figure 8. Experiment of solar ultraviolet radiation protective properties.

The yellowing of PU in all the samples shows that the UV resistance of PU is weak, see Table 5. The surface yellowing of 1433R+PU coating and PU fabric is serious. The surface of PVC waterproof cloth also shows slight yellowing and hardening. The Oxford cloth did not change significantly in the sun. There was no obvious yellowing of different Tyvek models after 4 hours of sunlight exposure. According to the comparative analysis of the charts, it is known that yellowing or hardening of commonly used materials in the market by ultraviolet radiation will reduce the service life of materials, while Tyvek material has good ultraviolet resistance and weather resistance to natural climate.

Table 5. Result of solar ultraviolet radiation protective properties.

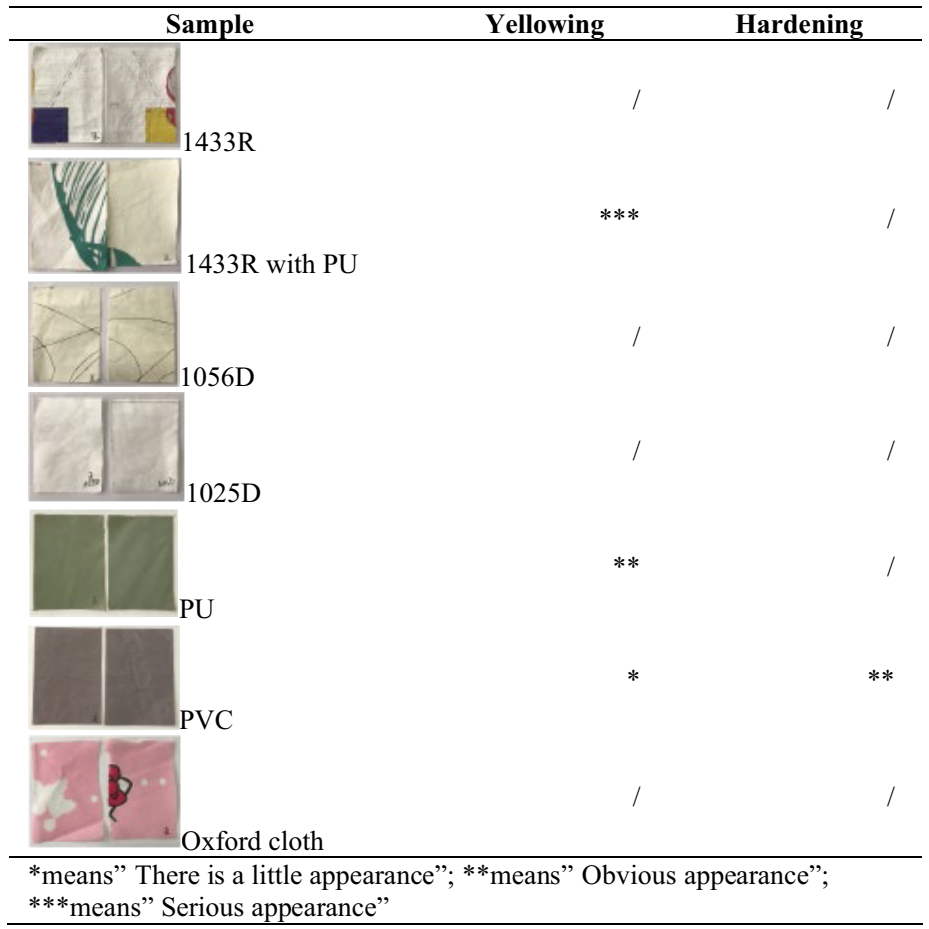




\section{Conclusion}

This paper aims to introduce Tyvek into the electric bicycles protection, and demonstrate its feasibility. Through the above material properties test, we found that Tyvek's performance is generally better than the existing materials in the market. The processed 1433R+PU contains PU, which shows aging phenomenon in the ultraviolet aging test. Clogging occurs during moisture permeability testing and is not suitable for windscreen design. Secondly, in the safety warning hot stamping process, 1025D and 1433R have melted, the melting point is lower than the processing temperature of hot stamping. Based on all the experimental results, Tyvek 1056D performs best in comfort and safety performance tests and is suitable for subsequent research on protective equipment design.

The various properties of Tyvek meet the criteria for EBs protective equipment material selection, and designers can classify materials by season based on their air permeability values. Moreover, the basic properties of Tyvek materials can be achieved without coating assistance, which reduces the processing flow of products. In a word, introducing Tyvek material into EBs protective equipment is a new attempt, which can greatly improve the service life of protective products and meet the safety and comfort needs of users. However, in future research, we need to combine the material characteristics of Tyvek (especially 1056D) with the specific characteristics of protective articles to provide more innovative possibilities for EBs product design.

\section{References}

[1] Citron, R.J.G. Electric bicycles: Li-Ion and SLA E-Bikes: Drivetrain, Motor, and Battery Technology Trends, Competitive Landscape, and Global Market Forecasts. Navigant Research, Boulder, CO, USA; 2016.

[2] Hung N B, Lim O. A review of history, development, design and research of electric bicycles[J]. Applied Energy, 2020, 260: 114323.

[3] Bernard TE, Luecke CL, Schwartz SW, Kirkland KS, Ashley CD. WBGT clothing adjustments for four clothing ensembles under three relative humidity levels. J Occup Environ Hyg. 2005;2(5):251-256.

[4] Xuemei J, Qiong Y, Ya W, et al. Effects of DuPont Tyvek ${ }^{\circledR}$ non-woven material mulching on fruit quality and chlorophyll fluorescence in Wanzhou Rose Orange[J]. Scientia Horticulturae, 2017, 219: 3136.

[5] Maslow A H. A theory of human motivation[J]. Psychological review, 1943, 50(4): 370.

[6] Seeley E S. The implications of Maslow's theory of motivation for consumer behavior: An hierarchical consumption theory[D]. New York University, Graduate School of Business Administration, 1988.

[7] ISO 9237, "Textiles-Determination of the permeability of fabrics to air" (International Standards Organization, Geneva, Switzerland, 1995).

[8] FZ/T 01149-2019, "Textiles-Evaluation for windproof and water-vapour" (ministry of industry and information technology of the People's Republic of China,2019)

[9] GB/T 21980-2017, "General technical specification for sportswear and protecting suits" (General Administration of Quality Supervision, Inspection and Quarantine of the People's Republic of China, 2017) 